\title{
Uso concurrente de elementos gráficos para potenciar la identificación tipológica de un producto a través del envase: estudio de caso en los refrescos de cola
}

\author{
Elementu grafikoen baterako erabilera, ontziaren bidez produktu baten
} identifikazio tipologikoa sustatzeko: kasuen azterketa kola-freskagarrietan

The concurrent use of graphic elements to strengthen a typological identification of a product through its container:

a case study with cola soft-drinks

\author{
Jordi Colet Ruz`, Ana Isabel Entenza Rodríguez \\ Universidad Autónoma de Barcelona
}

\begin{abstract}
RESUMEN: En el proceso de reconocimiento y selección de productos en los lineales del punto de venta es esencial identificar eficazmente las diferentes tipologías de producto para evitar confusiones molestas o, incluso, graves (personas diabéticas, por ejemplo). En este contexto, la función de la representación visual es fundamental. La presente investigación, de carácter cuasiexperimental, profundiza sobre esta cuestión y aborda la concurrencia de un texto y de un marcador cromático en el diseño del envase. Dicha circunstancia incrementaría la capacidad de identificación tipológica de los productos cuando se ubican en el punto de venta.
\end{abstract}

PALABRAS CLAVE: Diseño; imagen corporativa; comunicación; envase.

ABSTRACT: During the process of recognizing and selecting products on display shelves, it is essential to be able to identify efficiently and effectively different typologies of products to avoid any inconvenience or any serious confusion (specially, diabetics). In this context, the role of visual representation is fundamental. This almost-experimental research highlights insight into this issue and addresses the concurrence of graphic elements, as for instance a text with a chromatic marker, in containers' design. Such circumstance would increase a product typological identification capacity when they are placed at selling points.

KEYWORDS: Design; corporate image; communication; packaging.

\footnotetext{
* Correspondencia a / Corresponding author:: Jordi Colet Ruz. Universidad Autónoma de Barcelona, Edificio I, Campus de Bellaterra, Carrer de la Vinya, 08193 Cerdanyola del Vallès (Barcelona) - Jordi.Colet@uab.cat - http://orcid.org/0000-0002-0416-9255

Cómo citar / How to cite: Colet Ruz, Jordi; Entenza Rodríguez, Ana Isabel (2019). «Uso concurrente de elementos gráficos para potenciar la identificación tipológica de un producto a través del envase: estudio de caso en los refrescos de cola»; Zer, 24(46), 53-69. (https://doi. org/10.1387/zer.20265).
}

Recibido: 4 octubre, 2018; Aceptado: 3 diciembre, 2018.

ISSN 1137-1102 - eISSN 1989-631X / (c) 2019 UPV/EHU

(c) Esta obra está bajo una licencia 


\section{Introducción}

En marzo de 2015 los responsables corporativos de Coca-Cola ${ }^{\circledR}$ presentaron en España una identidad visual para sus envases fundamentada en la estrategia de unidad de marca ${ }^{1}$ : se unificaron las variedades del producto bajo una misma identidad $^{2}$, destacando logotipo y color rojo, pero dotando a cada tipología de producto de marcadores de color y textos identificadores (figura $1 \mathrm{a}$ y $1 \mathrm{~b}$ ).

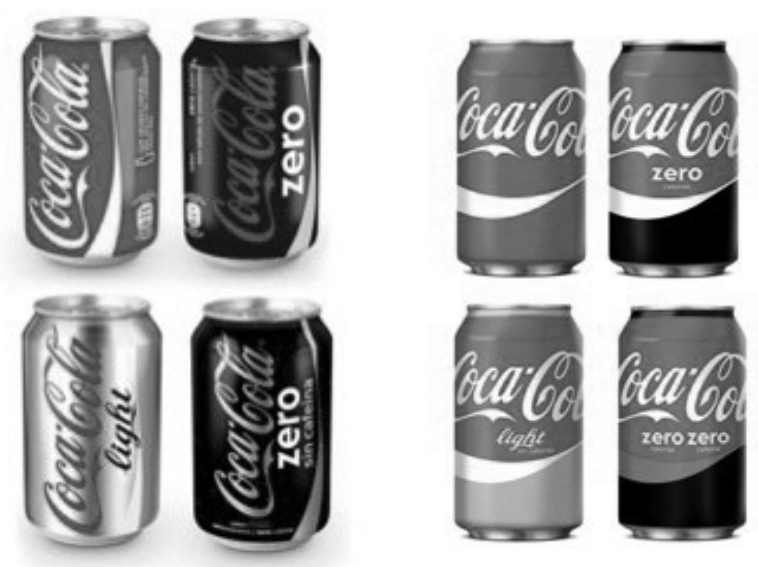

Fuente: http://brandemia.org/sites/default/files/sites/default/files/coca_cola_latas_antes_1.jpg y http://brandemia.org/sites/default/files/sites/default/files/coca_cola_despues.jpg [Consultada en junio 2016]

Figura $1 \mathrm{a}$ y $1 \mathrm{~b}$

Antes y después del rediseño de la identidad visual de Coca-Cola como marca única

Como podemos ver (figura 1b), en la nueva propuesta domina el color y el logotipo de la marca, mientras que se especifican las características de cafeína y azúcar de los productos mediante el uso codificado ${ }^{3}$ del marcador de color y texto identificadores ${ }^{4}$. Pero estos marcadores y textos no concurren, sino que ocupan posiciones diferentes.

\footnotetext{
1 Nota de prensa publicada en los principales diarios españoles en marzo de 2015 La fuente consultada, en http://www.cocacolaespana.es/historias/anuncio-Coca-Cola-marca-unica [consultada en junio de 2016]

2 Entendemos que la importancia de reforzar la marca se debe a que ha dejado de ser la más valiosa del planeta y ahora es tercera, tras Apple y Google (El Publicista, núm. 339, 2016, p. 11.)

3 Volveremos más adelante con el término, pero diremos aquí que nos referimos a código como lenguaje visual (Acaso, 2006: 24).

${ }^{4}$ Lo explicaremos más adelante, pero para esta investigación no es importante qué color o qué texto se utilice en la identificación, ya que nos interesan ambos como formas, como figuras que destacan sobre el fondo que se convierten en forma gracias a la memoria (Grupo $\mu$, 1993: 59-60; Dember y Warm, 1990: 261).
} 
En el momento de preparar este artículo, la marca presenta en España una nueva propuesta de diseño en la que cambian varias cosas (figura 2):

1. Concurren marcador de color y texto identificadores;

2. El texto y el marcador de color identificadores cambian la posición, y pasan de una posición centrada e inferior (figura 1 b) a una superior (figura 2);

3. Se modifica la forma o contorno ${ }^{5}$ del marcador de color identificador del producto;

4. La tipografia del texto identificador se unifica para todos los casos.

Estos cambios buscan mejorar la visibilidad de las variedades de refresco, y «facilitar las posibilidades de elección del consumidor para que escoja aquella Coca-Cola que mejor se adapte a sus necesidades y estilo de vida» ${ }^{6}$. Desconocemos el motivo de estos cambios, pero han implementado una solución gráfica que puede dar valor heurístico al presente trabajo, ya que puede ser útil si se constata que la concurrencia de texto y marcador de color deviene una circunstancia que facilita la identificación tipológica del producto.
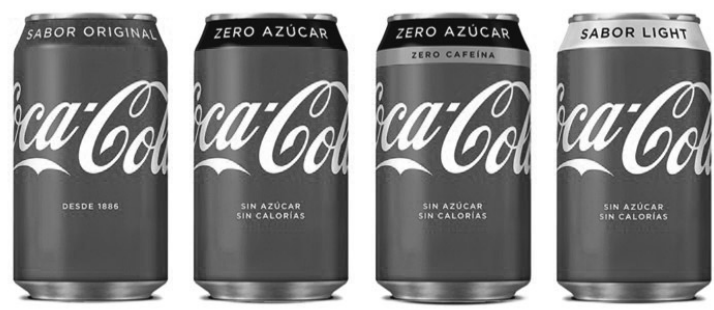

Fuente: https://www.cocacolaespana.es/historias/coca-cola-nuevo-diseno-envases [consultado el 3 de abril de 2018]

FiguRa 2

Nueva propuesta de imagen de la tipología de productos de CocaCola.

En estas páginas enfrentaremos las estrategias de «una única marca armónica multiproducto (...) en pro de construir una única marca en el mercado, más fuerte y reconocible.... $»^{7}$, utilizando en modo concurrente, y en el no concurrente, el marcador de color y el texto que identifican la tipología de producto.

5 Describiremos más adelante a qué nos referimos con este término, pero indicamos aquí que hacemos referencia a los límites, al contorno.

${ }^{6}$ Fuente https://www.cocacolaespana.es/historias/coca-cola-nuevo-diseno-envases [consultado el 3 de abril de 2018].

7 En http://www.elpublicista.es/anunciantes/coca-cola-inicia-nueva-etapa-taste-the-feeling [consultada en febrero de 2017]. 


\section{Objeto de estudio}

La presente investigación tiene por objeto estudiar el uso concurrente de marcador cromático y texto, como identificadores de una determinada tipología de refresco de cola; en particular, trata de demostrar que el uso concurrente de ambos - frente al uso simultáneo, pero no concurrente- potencia la identificación tipológica. Para ello se implementó el estudio con un envase de refresco de cola zero ${ }^{8}$.

\subsection{Hipótesis DE INVESTIGACIÓN}

Consideramos que cuando, en el código visual, coinciden en un mismo lugar (concurrencia) el texto y el marcador de color identificadores de un producto (el marcador de color en negro y el texto zero), se favorece la identificación y mejora el reconocimiento tipológico del producto. En base a lo expuesto, se detalla las siguientes hipótesis de investigación:

h1. Cuando el texto y el marcador de color, relativos a la tipología de producto, concurren en el envase, se incrementa la capacidad de identificación tipológica del mismo.

h2. La posición de la forma concurrente en el envase (superior, central o inferior) tiene efectos positivos sobre la capacidad de identificación del producto.

\section{Fundamentación}

\subsection{El lenguaje visual como código}

La comunicación visual es un sistema de transmisión de señales en el que utilizamos el lenguaje visual ${ }^{9}$ como codificador (Acaso, 2006: 24), entendiendo código como la competencia que limita «las posibilidades de combinación de los ele-

\footnotetext{
8 Se realizó un pretest en noviembre de 2016 entre alumnos de la Facultad de Ciencias de la Comunicación de la Universidad Autónoma de Barcelona. En él planteamos contemplar separadamente el estudio de las variedades de cola light y zero. Los resultados indicaron un mismo patrón de respuesta, independientemente de la variedad del producto, por lo que se decidió acotar el estudio en una única tipología.

9 Hemos utilizado este término de María Acaso porque, dado que casi cada autor propone su terminología: Eco, código visual; Saint Martin, lenguaje visual; Joly, mensaje visual; Grupo $\mu$, signo visual; Aumont y Villafañe, imágenes, etc., consideramos que la autora logra dar coherencia a todos ellos cuando establece la relación entre comunicación visual, lenguaje visual, mensaje visual e imagen (Acaso, 2006).
} 
mentos en juego, y el número de los que constituyen el repertorio» (Eco, 1986: 45), y que permite que el receptor comprenda el mensaje.

Mediante el código elaboramos una serie de unidades, las representaciones visuales (Acaso, op. cit., 31 y 37) que serán funcionales (véase Moles, 1990: 9) y comerciales, ya que su función principal es la venta de un producto o servicio (Acaso, op. cit. 118)

Respecto al código visual hemos de decir que, aunque la estrecha codificación del lenguaje verbal hace que otros sistemas parezcan poco codificados (Grupo $\mu$, 1993: 53), sus unidades transmiten conocimiento porque son vehículos «que alguien utiliza para algo» ${ }^{10}$ (Acaso, op. cit., 20), y que podemos comprender, aunque no conozcamos su gramática (Tusón, 1984: 143-144).

Sin embargo, como no podemos hablar de código genérico (Eco, op. cit., 268269), diremos que el código visual que nos ocupa corresponde a la tipología de productos de cola, en un momento determinado; un código que identifica cada producto mediante colores, textos identificadores y posición.

\subsection{LAS HERRAMIENTAS DEL LENGUAJE VISUAL: LA POSICIÓN Y LA FORMA (TEXTO Y MARCADOR DE COLOR)}

Aunque hay diversos tipos de articulación (Eco, op. cit., 203-206), tanto la palabra /casa/ como una bandera roja pueden ser analizados a partir de los elementos que los forman (Eco, 2000: 288), luego el código visual está formado por elementos. En este caso, y dada la variedad terminológica ${ }^{11}$, utilizaremos el término herramientas porque lo consideramos más neutro (no denota ni connota significación) y preciso que otros (elementos, variables), dada su función de construir representaciones visuales.

Aunque los autores consultados están de acuerdo en que una serie de herramientas construyen las representaciones visuales ${ }^{12}$ pero no coinciden al clasificarlas (Entenza, 2008), aquí concretaremos dos de ellas: la posición, como categoría topológica (Polidoro, 2016: 112), y la forma, como contorno identificador de texto y color.

10 Cursiva de la autora.

11 Los autores consultados no se ponen de acuerdo en la nomenclatura: para Villafañe, Wong, Dondis son elementos geométricos; fenómenos y elementos formales para Lupton y Cole; para Barthes, Joly, Grupo $\mu$, Sönesson, signos icónicos codificados y no codificados; para Saint Martin son variables visuales, herramientas para Acaso, categorías para Bañuelos, etc.

12 En cursiva la terminología que utilizaremos en el artículo. 
Así, consideraremos el texto como la forma que representa el lenguaje verbal, la forma de los sonidos (Valdés de León, 2012: 66-67; Joly 1994: 129), no como valores tipográficos o propiedades comunicativas. Y consideraremos el color como el fenómeno que determina los límites (Arnheim, 2008: 337), el elemento que posibilita la percepción de la forma (Caivano, 2005: 128), como lo que nos permite, antes que nada, distinguir formas, objetos e, incluso, cuantificarlos (Morgado, 2012: 106), pero no por sus propiedades o clasificación ${ }^{13}$.

Hemos de señalar que los autores consultados ${ }^{14}$ consideran la forma como una herramienta del lenguaje visual; y, respecto a la posición, aunque no hay unanimidad, varios autores hablan de posición como elemento (Wong), de variable del estado estético (Tena), de la gravedad y sus tensiones (Arnheim), del plano (Villafañe), de la composición (Joly, Parini), de la implantación en el plano (Saint Martin), de categorías topológicas (Polidoro), aspectos que podemos relacionar con la posición en el plano.

Finalmente, si oponemos forma y posición en el sentido lingüístico, y seguimos el criterio de propiedades independientes de Arnheim (op. cit., 337-338), comprobamos que la forma describe unos aspectos (redondez, angularidad) y la posición, otros (arriba, abajo, central, periférico).

\subsection{FORMA Y POSICIÓN}

\subsubsection{Forma}

Aunque algunas propuestas semióticas incorporan términos como categorías eidéticas para evitar ambigüedades con el término forma (Polidoro, op. cit., 108), creemos que podemos utilizarlo siempre que maticemos en qué sentido lo hacemos: nos referiremos con forma al contorno, al elemento que nos permite reconocer un objeto (Tatarkiewicz, 2004), a los límites exteriores del material visual (Acaso, op. cit., 54) que es centro de nuestra atención, y que destaca respecto de lo que le rodea (Lillo, 1993: 48-52; Grupo $\mu$, op. cit., 59).

En nuestro estudio nos centraremos en dos variaciones de la forma: la del marcador de color y la del texto que identifican la presencia de azúcar en el producto.

\footnotetext{
13 No nos interesa estudiar si un color forma parte, o no, de los 39 nuevos colores descritos para el lenguaje de las máquinas, en Lu Yu, Lichao Zhang, Joost van de Weijer, Fahad Shahbaz Khan, Yongmei Cheng, C. Alejandro Parraga. «Beyond Eleven Color Names for Image Understanding». Machine Vision and Applications 29 (2): 361-373, 2018, citado en https://www.agenciasinc.es/Noticias/Nuevos-nombrespara-describir-mejor-los-colores [Consultado en julio 2018].

14 Villafañe (2006), Wong (1986), Dondis (1992), Barthes (2002), Joly (1994), Grupo $\mu$ (1993), Sönesson (1992b), Saint Martin (1994), Tena (2004), Acaso (2006) y Leborg (2013).
} 
Consideramos el marcador de color identificador como forma porque nos referimos al material visual que aísla un contorno, el límite exterior que centra nuestra atención y se diferencia del fondo. Volveremos enseguida con esta cuestión de forma (figura) y fondo.

Y tratamos el texto como forma porque consideramos que la escritura es la forma que representa visualmente el lenguaje verbal, los sonidos de la lengua (Valdés de León, op. cit., 67), por lo que la imagen de las palabras posee dimensión completa (Joly, op. cit., 129) y forma parte del universo visual (Valdés de León, op. cit., 66-67), sin olvidar que «la matriz histórica de la escritura se halla en la expresión icónica» (Gubern, 1994: 59). Como hemos señalado en el apartado anterior, no valoraremos la potencia expresiva de las tipografias utilizadas.

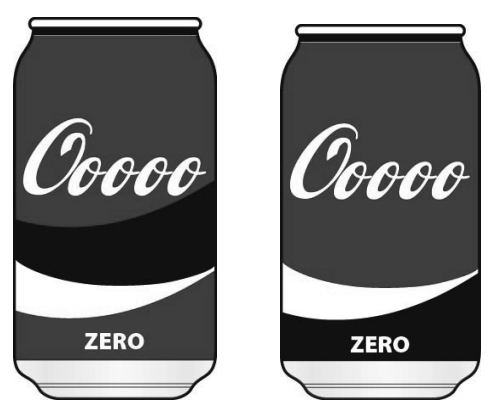

Fuente: elaboración propia.

Figura 3 a y b

Las dos formas identificadoras del producto no concurrentes, y concurrentes: la forma y el fondo

Las dos variantes de la forma que identifican el producto (marcador de color y texto, figura $3 \mathrm{a}$ ), son dos figuras sobre un fondo cuando no concurren, pero mantienen una relación de figura/fondo cuando el marcador de color y texto identificadores concurren (figura $3 b$ ). Vamos a detenernos un momento sobre figura, forma y fondo.

Denominamos figura, siguiendo la terminología de la Gestalt, a aquello que sometemos a atención, que aparentemente está delante, o encima, de lo que denominamos fondo (Grupo $\mu$, op. cit., 59; Saint Martin, 1994: 96-97; Lillo, op. cit., 386-387), que va a ser percibida como una cosa de bordes sólidos y compactos (Polidoro, op. cit., 91) y que reconocemos porque, habitualmente, tiene un tamaño más pequeño, y está incluida por el incluyente (fondo) (Polidoro, op. cit., 92). Por otra parte, hablaremos de forma para denominar la figura que convertimos en objeto (marcador, texto) gracias a la memoria (Grupo $\mu$, op. cit., 59-60; Dember y Warm, 1990: 261), y que es una de las herramientas del lenguaje visual. 
Si observamos las figuras $3 \mathrm{a}$ y $3 \mathrm{~b}$, veremos que en la primera sometemos a atención varias figuras objeto de nuestra atención, de las cuales nos interesan dos: marcador de color y texto identificadores de producto; en cambio, en la figura $3 b$, en la que concurren texto y marcador de color identificadores, tenemos una figura que emerge, que percibimos como objeto, que atrae nuestra atención: se trata de un modelo compacto, una forma global, una buena forma gestáltica que se impone como objeto en relación al fondo por su orientación, tamaño relativo, inclusión, u otros, y que requiere de menos información para ser percibida (Polidoro, op. cit., 92-96), a la que denominamos forma concurrente.

\subsubsection{Posición}

Las categorías topológicas describen la posición (alto, bajo, central, periférico) y la orientación (arriba/abajo, izquierda/derecha) de los elementos que están en el espacio bidimensional (Polidoro, op. cit., 112). En nuestro caso utilizaremos la descripción de la posición, pero no tendremos en cuenta la orientación.

En cuanto al espacio bidimensional, hemos elaborado representaciones bidimensionales de soportes tridimensionales, porque la opción bidimensional facilita la reproducción y el acceso a los materiales.

En este estudio analizaremos la posición desde dos puntos de vista: desde la concurrencia, esto es, cuando el texto y el marcador de color identificadores concurren, o no, en una misma posición, independientemente de cuál sea ésta; por otra parte, la posición alto, bajo y central cuando concurren el texto y el marcador de color identificadores, esto es, de la forma concurrente de ambos.

\subsection{DesCripCión DE ELEMENTOS IDENTIFICADORES SEGÚN EL CÓdigo TIPOLÓGICO DE LA MARCA LÍDER}

Nuestro estudio analizará qué disposición de herramientas codificadas (texto y marcador de color) facilita la identificación de un refresco de cola. Para desarrollar los prototipos hemos tomado como referencia el código identificador de CocaCola ${ }^{15}$, código en el que, a partir del uso fijo del color corporativo y del logotipo,

15 Desde una perspectiva histórica o de liderazgo, muchas marcas han experimentado cómo determinados elementos de la identidad gráfica de sus envases han devenido referentes de carácter tipológico. De hecho, la condición de marca pionera en una categoría de producto puede ser una excelente oportunidad para desarrollar una embrionaria codificación tipológica (Colet: 2013, 60). En este caso utilizaremos la codificación visual propuesta en España en marzo de 2015 y que se ha venido utilizando hasta principios de 2018. 
se posicionan texto y marcador de color identificadores, con diferentes funciones (véase figura 4):

a) marcador de ancho variable (forma ondulada) que cruza transversalmente el envase, aproximadamente a un tercio de su base. Tiene tres funciones en el código: reforzar la imagen de marca (trazo de estilo similar al del logotipo); identificar el producto con cafeína o sin cafeína (mediante color codificado); y es el límite para el color que identifique el nivel de azúcar.

b) En cuanto a los colores, unos refuerzan la imagen de marca (corporativos blanco y rojo), y otros indican el contenido de cafeína y azúcar (dorado, sin cafeína; gris, poco azúcar; negro, cero calorías).

c) En la parte inferior del envase (posición), se dispone un marcador de ancho variable con el color codificado del endulzamiento del producto: rojo corporativo para la azucarada, el gris para la light y el negro para la zero.

d) En la parte central (posición) del envase se disponen los términos light, zero, etc,.

e) $\mathrm{Y}$ en la parte superior (posición) tenemos una pequeña franja con el color de cada tipología de producto: rojo, negro o gris.



Fuente: http://www.elpoderdelasideas.com/wp-content/uploads/coca-cola-lata-espana-3.jpg [junio 2016]

FIgURA 4

Articulación del uso de los diferentes elementos formales en la marca y submarcas 
En nuestro caso hemos elaborado un prototipo experimental que reproduce aspectos del producto de zero calorías de la marca líder como, por ejemplo, el fondo de color continuo (en nuestro caso es azul para evitar una identificación total con la marca líder), una franja blanca transversal, que determina el límite de color identificador negro en dos de las posiciones, y dos marcadores de color negro que identifican el nivel de azúcar: una ondulada, que variará en función de la concurrencia y posición, y otra, recta, en la parte superior.

\section{Metodología}

En esta investigación, la metodología de trabajo implementada tiene carácter cuasiexperimental dado que se ha procedido a la manipulación y control de las variables objeto de estudio. Cada variable independiente remite a una propiedad específica del artefacto visual. En particular, la posición del texto y la del marcador de color relativos a la tipología de producto han sido los factores a partir de los cuales se han podido establecer los diferentes tratamientos experimentales.

La primera variable experimental (posición_texto) corresponde a la ubicación de la palabra ZERO en el envase; la segunda (posición_marcador color), corresponde a la ubicación del marcador de color indicativo de la tipología de producto.

Con dichas variables y sus respectivos valores, esta investigación desarrolla un diseño factorial de medidas repetidas (with-subject design). La prueba experimental implementada consta de 9 tratamientos visuales. Todas las propuestas remitían a la identidad visual de un refresco de cola con zero calorías. Dichos tratamientos son fruto de una determinada combinación establecida a partir de los factores y niveles experimentales. Con este procedimiento estadístico se ha planteado la manipulación simultánea de dos variables independientes y, consiguientemente, se han podido analizar dos cuestiones de interés: la influencia que cada variable ejerce sobre la variable dependiente y, al mismo tiempo, determinados efectos de interacción detectados entre las propias variables independientes. Por tanto, permite la valoración de los efectos principales (Igartua, 2006: 360) que cada factor provoca en la variable de estudio, pero considerando, también, la situación puntual del resto de los factores manipulados en el experimento (efectos de interacción).

Asimismo — al tratarse de un diseño experimental de medidas repetidas - un único grupo de sujetos es expuesto a la totalidad de los estímulos visuales, lo cual proporciona, por un lado, un mayor control experimental dado que la varianza entre grupos no se plantea y, por el otro, incrementa la potencia estadística de los resultados.

Para asegurar la validez interna de esta investigación, se procede al control de dos cuestiones relacionadas con el diseño experimental para evitar una contamina- 
ción de los resultados. La primera, relativa a la constitución de grupos homogéneos de sujetos. Para la realización de los experimentos se partió de una audiencia cautiva. Los participantes han formado parte de una muestra de conveniencia integrada por estudiantes universitarios y, también, por alumnos de ciclos formativos de grado superior. En ambos casos, se puede considerar que se trata de grupos homogéneos en aspectos como el nivel educativo, habilidades de lecto-escritura y familiaridad con entornos informáticos (Hampton, 1998). La segunda, relativa a las respuestas manifestadas por los sujetos las cuales podrían quedar condicionadas por el orden en que se exponen los estímulos. Para ello se establece una secuencia aleatoria para la exposición de las diferentes formulaciones visuales.

Por otra parte, la extensión de los resultados - la validez externa - puede interesar en investigaciones realizadas a partir de muestras representativas de una población. Sin embargo, este no es el principal objetivo de la presente investigación dado que se pretende el contraste de hipótesis causales (Igartua, op. cit., 338).

El desarrollo de la prueba experimental se ha realizado mediante la configuración de un formulario elaborado a través de Google Forms ${ }^{\circledR}$. Mediante esta aplicación se ha procedido al montaje del test de recepción el cual presenta, por un lado, una proposición que debe responderse a partir de una escala Likert de cinco niveles de respuesta - para indicar el grado de acuerdo o desacuerdo con el enunciado$\mathrm{y}$, por el otro, los correspondientes estímulos visuales objeto de estudio (figura 5).

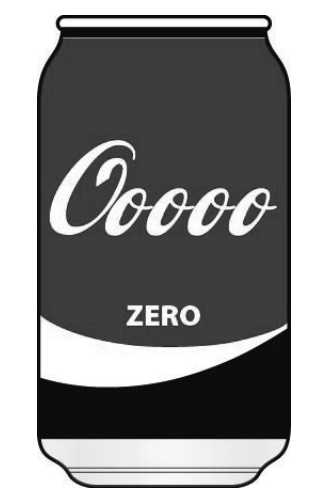

Fuente: elaboración propia.

Figura 5

Representación de uno de los prototipos experimentales

La proposición general que se planteó a los sujetos fue la siguiente:

Este envase permite una fácil identificación del refresco de cola zero. ¿Estás de acuerdo con este enunciado? 
Según la hipótesis de trabajo, las pruebas experimentales persiguen contrastar los efectos receptivos sobre los sujetos en relación a la facilidad para identificar un producto cuando el texto - representado por la palabra ZERO- y el del marcador de color — asociado al código cromático del producto- interactúan en la misma posición dentro del espacio gráfico (concurren).

En el experimento se plantea un diseño factorial (3) $\mathrm{x}(3)$ en el cual un único grupo de sujetos se expone a un conjunto de 9 tratamientos experimentales. La primera variable (posición_texto) tiene 3 niveles y la segunda (posición_marcador color) también dispone de 3 niveles. La exposición del grupo a la totalidad de los tratamientos permite la recogida de datos para su posterior análisis estadístico.

A continuación, se detalla la información correspondiente al experimento que se han realizado sobre la tipología de refresco de cola sin calorías:

- Producto: Refresco de cola zero.

- Variable dependiente: identificación visual.

- Factores y niveles:

- Posición_Texto

Valores nominales: 1. Superior; 2. Central; 3. Inferior

- Posición_Marcador Color

Valores nominales: 1. Superior; 2. Central; 3. Inferior

\subsection{DesCripción DE LA MUESTRA}

Respuestas válidas: 33

Edad: 32 sujetos entre 18 y 25 años; 1 sujeto entre 26 y 35 años.

Sexo: 8 hombres y 25 mujeres.

Estudios: 1 sujeto de ciclos formativos de grado superior y 32 sujetos de grado universitario.

\section{Resultados}

De acuerdo con los datos obtenidos en ambos experimentos, se procedió a analizar, por una parte, la fiabilidad del instrumento de medida y, por la otra, el nivel de significación (p) alcanzado en las pruebas de contraste estadístico con la aplicación SPSS. 


\subsection{Nivel DE FIABILIDAD Y SIGNIFICACIÓN}

De acuerdo con el coeficiente Alfa de Cronbach, los resultados indican un valor alto $(0,918)$ sobre la fiabilidad del instrumento de medida.

Con respecto al nivel de significación (p), la prueba de contraste indica que los efectos principales de los factores implicados en el estudio presentan en todos los casos valores $\leq 0,05$ (véanse tablas 1 y 2); en consecuencia, se puede concluir que se detectan diferencias significativas entre los niveles de las variables. Sin embargo, lo más relevante en la presente investigación es el efecto de interacción; en particular, la significación estadística alcanzada por la interacción entre los factores Posición_ Texto y Posición_Marcador Color, cuyo valor es $\mathrm{p}=0,001$ (véase tabla 3 ). Con dicho resultado se dan las condiciones para rechazar la hipótesis nula y, por tanto, se puede mantener la credibilidad de dicha hipótesis.

\section{TABLA 1}

Valores de significación de los contrastes correspondientes al factor POSICIÓN_TEXTO

\begin{tabular}{|c|c|c|c|c|c|}
\hline \multicolumn{7}{|c|}{ POSICIÓN TEXTO } \\
\hline Pruebas multivariante & Valor & F & $\begin{array}{c}\text { Gl de la } \\
\text { hipótesis }\end{array}$ & Gl del error & Sig. \\
\hline Traza de Pillai & 0,339 & 7,942 & 2,000 & 31,000 & 0,002 \\
\hline Lambda de Wilks & 0,661 & 7,942 & 2,000 & 31,000 & 0,002 \\
\hline Traza de Hotelling & 0,512 & 7,942 & 2,000 & 31,000 & 0,002 \\
\hline Raíz mayor de Roy & 0,512 & 7,942 & 2,000 & 31,000 & 0,002 \\
\hline
\end{tabular}

Fuente: Elaboración propia.

TABLA 2

Valores de significación de los contrastes correspondientes al factor POSICIÓN_MARCADOR_COLOR

\begin{tabular}{|c|c|c|c|c|c|}
\hline \multicolumn{7}{|c|}{ POSICIÓN MARCADOR COLOR } \\
\hline Pruebas multivariante & Valor & F & $\begin{array}{c}\text { Gl de la } \\
\text { hipótesis }\end{array}$ & Gl del error & Sig. \\
\hline Traza de Pillai & 0,586 & 21,959 & 2,000 & 31,000 & 0,000 \\
\hline Lambda de Wilks & 0,414 & 21,959 & 2,000 & 31,000 & 0,000 \\
\hline Traza de Hotelling & 1,417 & 21,959 & 2,000 & 31,000 & 0,000 \\
\hline Raíz mayor de Roy & 1,417 & 21,959 & 2,000 & 31,000 & 0,000 \\
\hline
\end{tabular}

Fuente: Elaboración propia. 
TABLA 3

Valores de significación de los contrastes relativos a la interacción entre POSICIÓN_TEXTO y POSICIÓN_MARCADOR_COLOR

\begin{tabular}{|c|c|c|c|c|c|}
\hline \multicolumn{7}{|c|}{ POSICIÓN TEXTO ^ POSICIÓN MARCADOR COLOR } \\
\hline Pruebas multivariante & Valor & F & $\begin{array}{c}\text { Gl de la } \\
\text { hipótesis }\end{array}$ & Gl del error & Sig. \\
\hline Traza de Pillai & 0,460 & 6,172 & 4,000 & 29,000 & 0,001 \\
\hline Lambda de Wilks & 0,540 & 6,172 & 4,000 & 29,000 & 0,001 \\
\hline Traza de Hotelling & 0,851 & 6,172 & 4,000 & 29,000 & 0,001 \\
\hline Raíz mayor de Roy & 0,851 & 6,172 & 4,000 & 29,000 & 0,001 \\
\hline
\end{tabular}

Fuente: Elaboración propia.

\section{Conclusiones}

En la presente investigación partíamos de que la concurrencia del texto y el marcador de color en el mismo espacio gráfico era una circunstancia que podía incrementar la capacidad de identificación tipológica de un producto. Los resultados de los contrastes estadísticos realizados en la prueba experimental permiten aceptar la validez de la primera hipótesis. En particular, merece la pena destacar la relevancia que tienen los efectos de interacción, en todos los casos, entre la posición que adopta la forma ZERO con respecto a la posición del marcador cromático (negro). En consecuencia, se puede inferir que la concurrencia de ambos elementos gráficos es significativa a los efectos de identificación de un producto. Asimismo, las medias marginales estimadas han permitido determinar cuál ha sido la tendencia en el comportamiento de respuesta. Los resultados de la prueba experimental indican que la concurrencia entre la posición del texto (zero) y la del marcador cromático (negro) ha tenido efectos positivos para la identificación del producto por parte de los sujetos. En particular, cuando la concurrencia de la posición del texto (zero) y el marcador cromático (negro) se ubicaba preferentemente en la zona central del envase; con ello, daríamos por válida la segunda hipótesis.

En definitiva, el objetivo fundamental del trabajo ha sido contrastar la influencia de la posición concurrente de texto (zero) y marcador de color en aras de incrementar la capacidad de identificación tipológica de un producto. En este sentido, un mayor rendimiento comunicativo del envase debe tener la firme vocación de evitar confusiones o contratiempos a los consumidores, especialmente cuando las propiedades del producto pueden afectar a un determinado colectivo, como es el caso de las personas diabéticas. 
No obstante, la investigación adolece de limitaciones dado que se ha circunscrito al estudio de un determinado producto que, por su contenido habitual en azúcares, debe ser objeto de alerta para determinados consumidores. En el futuro, la investigación podría implementarse para otras tipologías de producto que puedan ser susceptibles de provocar otras intolerancias. En este sentido, los colectivos que presentan determinadas contraindicaciones para el consumo de algunos productos son más sensibles a este tipo de información y, por tanto, el uso de una muestra específica podría aportar mayor información al respecto.

\section{Referencias bibliográficas}

Acaso, María (2006) El lenguaje visual. Barcelona: Paidós Ibérica.

Albers, J. (1979) La interacción del color. Madrid: Alianza Forma.

Arnheim, R. (2008). Arte y percepción visual. 2. ${ }^{a}$ edición, 4. ${ }^{a}$ reimpresión. Madrid: Alianza editorial.

Bañuelos Capistrán, Jacob (2006). Aplicación de la semiótica a los procesos del diseño. UNED. Revista Signa 15 (2006), 233-254. doi: 10.5944/signa.vol15.2006.6134

Barthes, Roland (2002). Rhétorique de l'image. Oeuvres Completes, (pp. 573-588). Vol. II, Paris: Éditions du Seuil.

Caivano, José Luis (2005) Semiótica, cognición y comunicación visual: los signos básicos que construyen lo visible. Tópicos del seminario, v. 1, n. 13. Pp 113-135. http://www.topicosdelseminario.buap.mx/index.php/topsem/article/view/325/318

Colet Ruz, J. (2018) Comunicació visual a través del packaging. Barcelona: Edición autoeditada.

Cossette, Claude. (1983) Les Images démaquillées: approche scientifi que de la communication par l'image. Québec: Éditions Riguil.

Dember, William N. y Warm, Joel S. (1990) Psicología de la percepción. Madrid: Alianza Editorial.

Eco, U. (1986) La estructura ausente. 3. ${ }^{a}$ edición. Barcelona: Editorial Lumen. - (2000) Tratado de semiótica general. 5. ${ }^{a}$ edición. Barcelona: Editorial Lumen.

Dondis, D.A (2017) La sintaxis de la imagen. 2. ${ }^{a}$ edición 2. ${ }^{a}$ tirada. Barcelona: Gustavo Gili.

Entenza Rodríguez, A. I. (2008) Elementos básicos de las representaciones visuales funcionales. Análisis crítico de las aportaciones realizadas desde diversas disciplinas. [Tesis doctoral] Bellaterra: Universidad Autónoma de Barcelona. Disponible en http://www.tdx.cat/ handle/10803/299367

Grupo $\mu$ (1993) Tratado del signo visual. Madrid: Cátedra.

Gubern, R. (1994) La mirada opulenta. Barcelona: Gustavo Gili.

Hampton, J. (1998) The between-subjects experiment. Laboratory psychology. A beginner's guide. (pp.15-38). Hove: Psychology Press, 
Igartua, J. J. (2006) Métodos cuantitativos de investigación en comunicación. Barcelona: Editorial Bosch.

Joly, Martine. (1993) Introduction à l'analyse de l'image, París, Editions Nathan.

- (1994) L'image et les signes, París, Editions Nathan.

- (1999) Introducción al análisis de la imagen. Buenos Aires: La marca.

Luna, Dolores y Tudela, Pio (2016) Percepción visual. 2. ${ }^{a}$ edición. Madrid: Editorial Trotta.

Lupton, Ellen y Phillips, Jennifer Cole (2009) Diseño Gráfico: Nuevos fundamentos. Barcelona: Ediciones Gustavo Gili

Kalat, James W. (2004) Psicología biológica. 8. a edición. Madrid: Ediciones Paraninfo.

Klinkenberg, Jean-Marie (2005) La semiótica visual: grandes paradigmas y tendencias de línea dura. Tópicos del seminario, v. 1, n. 13. Pp. 19-47. http://www.topicosdel seminario.buap.mx/index.php/topsem/article/view/321/314

Kondratov, A.M. (1973) Del sonido al signo. Buenos Aires: Paidós.

Lillo Jover, Julio. (1993) Psicología de la percepción. Madrid: Debate.

Leborg, Christian (2013) Gramática visual, Barcelona, Editorial Gustavo Gili.

Moles, A.; Janiszewski, L. (1990) Grafismo Funcional. Barcelona: Ceac.

Barnés, Jorge Santiago (2006) La interdisciplinariedad de la imagen en el desarrollo de la narrativa visual. Razón y palabra, 49, p. 10

Morgado, Ignacio. (2012) Cómo percibimos el mundo. Una exploración de la mente y los sentidos. Barcelona: Editorial Ariel.

Polidoro, Piero. (2016) ¿Qué es la semiótica visual? Bilbao: Servicio editorial de la Universidad del País Vasco.

Saint Martin, F. (1994) Sémiologie du Langage Visuel. Québec: Presses de l'Université du Québec.

Sönesson, G. (1992) Comment le sens vient aux images. Pour un autre discours de la méthode, en Marie Carani Ed. De l'histoire de l'art à la sémiotique visuelle (pp. 29-83). Québec: Les éditions du Septentrion.

Tatarkiewicz, W. (2004) Historia de seis ideas. Madrid: Tecnos.

Tena Parera, Daniel. (2004) Diseño gráfico y comunicación. Madrid: Pearson educación

Tusón, Jesús. (1984) Lingüística. Una introducción al estudio del lenguaje, con textos comentados y ejercicios. Barcelona: Barcanova.

Villafañe, Justo. (2006) Introducción a la teoría de la imagen. Reimpresión. Madrid: Pirámide.

Valdés de León, Gustavo A. (2012) De la «Alfabetidad Visual» a la Semiótica. Otra aproximación al «Lenguaje» Visual». Cuad. Cent. Estud. Diseño Comun., Ens 39. pp. 57-69. http://www.scielo.org.ar/scielo.php?script=sci_arttext\&pid=S185335232012000100005

Wong, Wicius (1986) Fundamentos del diseño bi y tridimensional. 5. ${ }^{a}$ edición. Barcelona: Gustavo Gili.

Zeki, Semir (1985) Una visión del cerebro. Barcelona: Ariel. 


\section{REVISTAS ESPECIALIZADAS}

El Publicista. (2016). Madrid: Publicaciones Especializadas, 339.

\section{EN LÍNEA}

http://www.cocacolaespana.es/historias/anuncio-Coca-Cola-marca-unica [Consultada, junio 2016].

http://www.elpoderdelasideas.com/wp-content/uploads/coca-cola-lata-espana-3.jpg [Consultada, junio 2016].

http://brandemia.org/sites/default/files/sites/default/files/coca_cola_latas_antes_1.jpg [Consultada, junio 2016].

http://brandemia.org/sites/default/files/sites/default/files/coca_cola_despues.jpg [Consultada, junio 2016].

http://www.elpublicista.es/anunciantes/coca-cola-inicia-nueva-etapa-taste-the-feeling [Consultada, febrero 2017].

http://www.factoriacreativabarcelona.es/coca-cola-renueva-su-imagen/. [Consultada, febrero 2017].

http://www.europapress.es/economia/noticia-coca-cola-lanza-estrategia-marca-unicapermitira-acelerar-ventas-20160121172429.html [Consultada, febrero 2017].

http://www.guioteca.com/diabetes/coca-cola-cambia-diseno-de-sus-envases-molestia-endiabeticos-por-posible-confusion/ [Consultada, septiembre 2017].

http://www.cocacolaespana.es/historias/coca-cola-nuevo-diseno-envases [Consultada, 3 de abril 2018].

https://www.agenciasinc.es/Noticias/Nuevos-nombres-para-describir-mejor-los-colores, [Consultada, julio 2018]. 